\title{
Deleterious Effects of Calcium Channel Blockade on Pressure Transmission and Glomerular Injury in Rat Remnant Kidneys
}

\author{
Karen A. Griffin, Maria M. Picken, and Anil K. Bidani \\ Departments of Medicine and Pathology, Loyola University Medical Center and Hines Veterans' Administration Hospital, \\ Maywood, Illinois 60153
}

\begin{abstract}
Hypertensive mechanisms are postulated to play a major role in the progressive glomerulosclerosis (GS) after renal mass reduction. But, in contrast to converting enzyme inhibitors, BP reduction by calcium channel blockers, has not provided consistent protection. Radiotelemetric BP monitoring for 7 wk was used to compare nifedipine (N) and enalapril $(E)$ in the rat $\sim 5 / 6$ renal ablation model. After the first week, rats received $N, E$, or no treatment $(C)$. The overall averaged systolic $B P$ in $C(173 \pm 7 \mathrm{mmHg})$ was reduced by both $E$ and $N(P<0.001)$, but $E$ was more effective (113 \pm 2 vs. $134 \pm 3 \mathrm{mmHg}, P<0.01)$. GS was prevented by $E(2 \pm 1$ vs. $26 \pm 5 \%$ in $C)$ but not by $N(25 \pm 6 \%)$. GS correlated well with the overall averaged BP in individual animals of all groups, but the slope of the relationship was significantly steeper in $\mathbf{N}$ compared with $\mathbf{C}+\mathbf{E}$ rats $(P$ $<0.02$ ), suggesting greater pressure transmission to the glomeruli and GS for any given BP. Since autoregulatory mechanisms provide the primary protection against pressure transmission, renal autoregulation was examined at 3 wk in additional rats. Autoregulation was impaired in $\mathbf{C}$ rats, was not additionally altered by $E$, but was completely abolished by $\mathbf{N}$. These data demonstrate the importance of autoregulatory mechanisms in the pathogenesis of hypertensive injury and suggest that calcium channel blockers which adversely affect pressure transmission may not provide protection despite significant BP reduction. (J. Clin. Invest. 1995. 96:793-800.) Key words: hypertension - glomerulosclerosis, focal • nifedipine $\cdot$ enalapril $\bullet$ renal circulation
\end{abstract}

\section{Introduction}

The rat remnant kidney (RK) ${ }^{1}$ model (produced by right nephrectomy and segmental infarction of two-thirds of the left

This work was presented in part at the 27th Annual Meeting of the American Society of Nephrology in Orlando, FL on 27 October 1994 and was published in abstract form (1994. J. Am. Soc. Nephrol. 5:603).

Address correspondence to Karen A. Griffin, M.D., Loyola University Medical Center, Section of Renal Disease and Hypertension, 2160 South First Avenue, Maywood, IL 60153. Phone: 708-343-7200 × 4120; FAX: 708-531-7978.

Received for publication 24 February 1995 and accepted in revised form 25 April 1995.

1. Abbreviations used in this paper: $\mathrm{A}_{\mathrm{G}}$, cross-sectional area; $\mathrm{AI}$, autoregulatory index; $\mathrm{C}$, untreated controls; $\mathrm{CCB}$, calcium channel blockers; $E$, enalapril; $N$, nifedipine; $P_{G C}$, glomerular capillary pressure; $R B F$, renal blood flow; RK, remnant kidney; RPP, renal perfusion pressure; $\mathrm{S}_{\mathrm{Cr}}$, serum creatinine; $\mathrm{V}_{\mathrm{G}}$, glomerular volume.

The Journal of Clinical Investigation, Inc.

Volume 96, August 1995, 793-800 kidney) has been extensively used to investigate the progressive nature of human renal disease (1-8). It has been suggested that the compensatory increases in structure and/or function of remnant nephrons may be maladaptive as with time these RK rats develop a syndrome of hypertension, proteinuria, and progressive glomerulosclerosis $(3-7)$. The compensatory vasodilation of the remnant renal vasculature is postulated to result in an enhanced transmission of the elevated systemic pressures with resultant increases in glomerular capillary pressure $\left(\mathrm{P}_{\mathrm{GC}}\right)$ and consequent glomerular injury and sclerosis $(4,5,7-11)$. However, studies have shown that, despite equivalent BP control, different antihypertensive agents do not provide equivalent glomeruloprotection $(9,12-14)$. This has led to the suggestion that BP-independent mechanisms such as glomerular hypertrophy may be more important, both in the pathogenesis of glomerular injury and in the glomeruloprotection conferred by antihypertensive agents $(6,13,14)$. In the case of calcium channel blockers (CCB), both the extent and the mechanisms of glomeruloprotection remain disputed $(13,15-19)$. Differences have been noted not only between the different classes of CCB but even within the same class. The interpretations in these studies as to the pathogenetic role of hypertensive mechanisms are critically dependent on the adequacy of periodic tail-cuff BP measurements to accurately assess the differences in systemic BP within and between groups. Our recent studies using the radiotelemetric technique of continuous BP monitoring have demonstrated a marked spontaneous lability in the RK model $(20,21)$. Such lability severely limits the capacity of the tailcuff methodology to accurately assess the ambient systemic BP profiles and, therefore, to examine the relationship of glomeruloprotection to the relative antihypertensive effectiveness of antihypertensive regimens. The present studies examine the relationship of glomeruloprotection by the CCB, nifedipine, and the converting enzyme inhibitor, enalapril, to their effects on radiotelemetrically monitored systemic blood pressure, glomerular hypertrophy, and renal autoregulation in the RK model.

\section{Methods}

Two sets of investigations were performed on male Sprague-Dawley rats (body wt 200-300 grams) that were fed a standard protein diet (Purina, St. Louis, MO) and synchronized to a 12:12-h light (6:0018:00 h) and dark (18:00-6:00 h) cycle. All rats received food and water ad libitum throughout the study.

Radiotelemetry studies. The rats were anesthetized with sodium pentobarbital ( $45 \mathrm{mg} / \mathrm{kg}$ intraperitoneally) and subjected to $\sim 5 / 6$ renal ablation (right nephrectomy and ligation of all but one posterior extrarenal branch of the left renal artery) $(8,20,21)$. Rats were prepared for telemetric monitoring of blood pressure (Data Sciences, International, St. Paul, MN) at the time of the renal ablation surgery $(20,21)$. Each rat had a blood pressure sensor (model TA11PA-C40; Data Sciences International) inserted intraperitoneally. The sensor's catheter was inserted into the aorta below the level of the renal arteries, and the radio 
frequency transmitter was fixed to the peritoneum. The rats were housed individually in plastic cages that were placed on top of the receiver. The signals from the pressure sensor were converted, temperature compensated, and sent via the radio frequency transmitter to the telemetry receiver. The receiver was connected to a BCM-100 consolidation matrix which transmitted the information to the Dataquest IV acquisition system (Data Sciences International). Systolic blood pressure in each animal was continuously recorded at 10 -min intervals for the duration of the study. Tail vein blood samples were obtained at $3 \mathrm{~d}$ for measurement of serum creatinine $\left(\mathrm{S}_{\mathrm{Cr}}\right)$ as an index of the degree of renal mass reduction. At $\sim 7 \mathrm{~d}$, the rats were randomly assigned to receive either no treatment $(\mathrm{C})$, nifedipine $(\mathrm{N})(0.25 \%$ by weight in standard rat chow), or enalapril (E) ( $50 \mathrm{mg} /$ liter of drinking water). As nifedipine is photosensitive, the food was covered by aluminum foil to prevent photodegradation. The number of animals for each group in the radiotelemetry study were: $\mathrm{C}(n=12), \mathrm{E}(n=12)$, and $\mathrm{N}(n=16)$. These numbers do not include two controls and three nifedipine-treated rats that died during the course of the studies. After $7 \mathrm{wk}$, tail vein $\mathrm{S}_{\mathrm{Cr}}$ and 24-h urine collections for protein excretion were obtained. The rats were then anesthetized with intravenous sodium pentobarbital $(40 \mathrm{mg} / \mathrm{kg})$, a tracheostomy was performed using polyethylene (PE-200) tubing, and the rats were surgically prepared for clearance studies as described previously $(5,8)$. In brief, a carotid artery was cannulated with PE-50 tubing and connected to a Windograf (model 40-8474; Gould Inc., Glen Burnie, MD) for continuous recording of mean systemic blood pressure. A femoral vein was cannulated with PE-50 tubing, and a priming dose of inulin in $150 \mathrm{mM} \mathrm{NaCl}$ was administered, followed by a continuous maintenance infusion of $150 \mathrm{mM} \mathrm{NaCl}$ containing inulin at $0.055 \mathrm{ml} /$ min to maintain the plasma concentration of inulin at $\sim 50 \mathrm{mg} / \mathrm{dl}$ and for replacement of surgical and ongoing fluid losses. The left ureter was then cannulated with polyethylene tubing for collection of urine samples. A $1.0 \mathrm{mM}$ R series flow probe (Transonic Systems Inc., Ithaca, NY) was placed around the left renal artery for measurement of renal blood flow (RBF) by a flowmeter (Transonic Systems Inc.), as described previously $(22,23)$. At the conclusion of the surgery, a $150 \mathrm{mM} \mathrm{NaCl}$ bolus equal to $1 \%$ of body weight was administered. Two 20 -min clearances of inulin were obtained. Blood samples were obtained at the midpoint of each urine collection. At the conclusion of these studies the rats were killed, and the kidneys were harvested for morphometric and morphological studies.

Autoregulatory studies. Rats assigned to the autoregulatory studies underwent renal ablation and were followed for $3 \mathrm{wk}$ without radiotelemetry. Tail vein $\mathrm{S}_{\mathrm{Cr}}$ was obtained at $3 \mathrm{~d}$ to stratify for renal mass reduction. At $\sim 7 \mathrm{~d}$, the rats were randomly assigned to receive either no treatment $(C)$, nifedipine $(0.25 \%$ by weight in standard rat chow), or enalapril $(50 \mathrm{mg} / \mathrm{liter}$ of drinking water). The number of animals for each group in the autoregulatory study were: $\mathrm{C}(n=10), \mathrm{E}(n$ $=10)$, and $\mathrm{N}(n=10)$. After 3 wk the rats were anesthetized with intravenous sodium pentobarbital $(40 \mathrm{mg} / \mathrm{kg})$ and underwent clearance studies (see above). A $1.0 \mathrm{mM}$ R series flow probe (Transonic Systems Inc.) was placed around the left renal artery for measurement of RBF. Autoregulatory studies were performed using aortic miniclamps positioned above and below the left renal artery to raise or lower renal perfusion pressure (RPP) as previously described $(22,23)$. The RBF was allowed to stabilize for 1-2 $\mathrm{min}$ at each pressure before RBF measurements were made. At the conclusion of these studies the rats were killed, and the kidneys were harvested for morphometric and morphological studies.

Morphometric and morphologic studies. Transverse sections of the kidney through the papilla were fixed in situ by perfusion for $5 \mathrm{~min}$ at the measured blood pressure with $1.25 \%$ glutaraldehyde in $0.1 \mathrm{M}$ cacodylate buffer. Sections were cut at a thickness of $2 \mu \mathrm{m}$ and stained with hematoxylin and eosin, periodic acid-Schiff, and toluidine blue. Glomerular volume was measured by area perimeter analysis (Bioquant System IV software; R\&M Biometrics, Inc., Nashville, TN). The cross-sectional area $\left(A_{G}\right)$ of 75 consecutive glomerular profiles contained in one kidney section for each animal was measured using a digitizing pad as described previously $(24)$. The mean glomerular volume $\left(\mathrm{V}_{\mathrm{G}}\right)$ was then calculated from the respective mean $A_{G}$ as $V_{G}=\beta / \kappa\left(A_{G}{ }^{3 / 2}\right)$, where $\beta=1.38$ is the size distribution coefficient and $\kappa=1.1$ is the shape coefficient for glomeruli idealized as spheres (25). For the morphologic studies, sections were evaluated systematically in each kidney for glomerular injury (segmental sclerosis and/or necrosis) in a blinded fashion by standard morphologic methods. At least 100 glomeruli in each animal were evaluated, and the severity of glomerular injury was expressed as the percentage of glomeruli exhibiting such injury $(20,21)$.

Analyses, calculations, and statistics. Urinary protein was measured by the quantitative sulfosalicylic acid method with human serum albumin serving as standard. Serum creatinine was measured using a creatinine analyzer (Beckman Instruments, Inc., Fullerton, CA) $(20,21)$. Inulin in urine and plasma filtrates was determined spectrophotometrically by the diphenylamine method as described previously $(20,21)$. Glomerular filtration rate (GFR) was calculated using standard formulae. Autoregulatory index (AI) was calculated by the method of Semple and deWardener (26) as follows: $\mathrm{AI}=\left[\left(\mathrm{RBF}^{2}-\mathrm{RBF}^{1}\right) / \mathrm{RBF}^{1}\right] /$ $\left[\left(\mathrm{RPP}^{2}-\mathrm{RPP}^{1}\right) / \mathrm{RPP}^{1}\right]$. An $\mathrm{AI}$ of 0 indicates perfect autoregulation while an AI of 1 indicates the vessels act as passive conduits for blood flow. All results are expressed as mean \pm SE. Statistical analysis was performed using ANOVA followed by Student-Newman-Keuls test or by Kriskall-Wallis nonparametric ANOVA followed by Dunn's multiple comparison test as appropriate (27). A $P$ value of $<0.05$ was considered statistically significant.

\section{Results}

Radiotelemetry studies. Table I shows that the initial body weight, $\mathrm{S}_{\mathrm{Cr}}$, and 24-h urine protein excretion were not different between the three groups undergoing radiotelemetry studies. Similarly, the $\mathrm{S}_{\mathrm{Cr}}$ at $3 \mathrm{~d}$ was not different between the groups, indicating comparable renal mass reduction in all groups. The final body weight was significantly lower in the nifedipinetreated rats as compared with both the control and enalapriltreated rats, but no differences were noted for the final $\mathrm{S}_{\mathrm{Cr}}$, $\mathrm{RBF}$, and GFR between the three groups. Table I also provides the kidney weights and glomerular volume at $7 \mathrm{wk}$ after renal ablation for the three groups. Although kidney weight was significantly lower in the enalapril-treated rats as compared with the control and nifedipine-treated rats, there was no significant difference in glomerular volumes between the three groups.

Fig. 1 illustrates the course of 24-h averages of radiotelemetrically monitored systolic $\mathrm{BP}$ after $\sim 5 / 6$ renal ablation and the effects of the different antihypertensive agents over $7 \mathrm{wk}$. For the first $7 \mathrm{~d}$ before the initiation of nifedipine and enalapril therapy, the BP was similar in all three groups. Both nifedipine and enalapril reduced systemic BP as compared with the control RK rats, but enalapril was more effective in lowering BP than nifedipine. This is demonstrated by the significant differences between each of the three groups with respect to the means of the overall averaged systolic BP (the mean of all $\sim 6,000 \mathrm{BP}$ readings after day 7 ) (Fig. 2). Fig. 3, $a-c$, illustrates the course of telemetric systolic BP recordings at 10-min intervals in a rat from each of the groups. Fig. $3 a$ graphically displays the spontaneous fluctuations of systolic BP in an untreated rat. These spontaneous fluctuations persisted but at reduced levels in both nifedipine- and enalapril-treated rats. However, the reduction as indicated by the standard deviation of the overall averaged BP (Fig. 2) was statistically significant only for the enalapril-treated rats.

Fig. $4 a$ illustrates the final 24-h urine protein excretion and glomerular injury data at $7 \mathrm{wk}$ after renal ablation for the animals in the three groups that underwent radiotelemetry studies. Protein excretion was not different between untreated and nifed- 
Table I. Renal Functional and Structural Parameters for Radiotelemetry Studies

\begin{tabular}{|c|c|c|c|c|c|c|c|c|c|c|}
\hline & \multicolumn{3}{|c|}{ Initial } & \multirow{2}{*}{$\frac{3 \mathrm{~d}}{\mathrm{~s}_{\mathrm{cr}}}$} & \multicolumn{6}{|c|}{ Final (7 wk) } \\
\hline & Body wt & $\mathbf{S}_{\mathrm{cr}}$ & Proteinuria & & Body wt & $\mathrm{S}_{\mathrm{cr}}$ & GFR & $\mathrm{RBF}$ & Kidney wt & $\mathrm{v}_{\mathrm{G}}$ \\
\hline & grams & $m g / d l$ & $m g / 24 h$ & $m g / d l$ & grams & $m g / d l$ & $\mathrm{~m} / \mathrm{min} / \mathrm{kg}$ & $\mathrm{m} / \mathrm{min} / \mathrm{kg}$ & grams $/ \mathrm{kg}$ & $\mu m^{-3} \times 10^{-6}$ \\
\hline $\mathrm{C}(n=12)$ & $251 \pm 8.8$ & $0.40 \pm 0.02$ & $3.6 \pm 0.6$ & $0.84 \pm 0.06$ & $423 \pm 19^{\circ}$ & $0.91 \pm 0.08$ & $2.3 \pm 0.5$ & $28.9 \pm 2.2$ & $8.4 \pm 0.4$ & $2.8 \pm 0.2$ \\
\hline $\mathrm{E}(n=12)$ & $245 \pm 5.9$ & $0.39 \pm 0.02$ & $2.2 \pm 0.4$ & $0.78 \pm 0.04$ & $436 \pm 15^{*}$ & $0.76 \pm 0.06$ & $3.28 \pm 0.27$ & $36.4 \pm 2.4$ & $6.3 \pm 0.2^{* \ddagger}$ & $2.7 \pm 0.2$ \\
\hline $\mathrm{N}(n=16)$ & $249 \pm 2.9$ & $0.38 \pm 0.02$ & $2.9 \pm 0.5$ & $0.87 \pm 0.04$ & $359 \pm 15^{\ddagger}$ & $0.99 \pm 0.15$ & $2.43 \pm 0.35$ & $30.1 \pm 2.5$ & $7.9 \pm 0.3$ & $2.8 \pm 0.2$ \\
\hline
\end{tabular}

After baseline studies, all rats underwent $<5 / 6$ ablation and underwent radiotelemetric BP monitoring for 7 wk. After the first $7 \mathrm{~d}$, rats were left untreated (C) or received nifedipine $(N)$ or enalapril $(E)$. RBF and GFR data were not obtained for one rat each in the $C$ and $N$ groups. ${ }^{*} P<0.01$ vs. $\mathrm{N} ;{ }^{\ddagger} P<0.01$ vs. $\mathrm{C}$

ipine-treated rats but was significantly lower in the enalapriltreated rats $(P<0.01$ vs. $C$ and $N)$. Similarly, glomerular injury expressed as the percentage of glomeruli exhibiting lesions of segmental sclerosis and/or necrosis was not different between untreated and nifedipine-treated rats but was significantly reduced in the enalapril group $(P<0.001$ vs. $\mathrm{C}$ and $\mathrm{N})$.

Fig. 5 illustrates the relationship of the percentage of glomerular injury with the overall averaged systolic BP for each animal. Glomerular injury decreased with decreasing BP and the correlation coefficient $(r)$ for all animals was $0.57, P<0.0001$. However, as can be noted, the $\mathrm{N}$ rats had less of a reduction in glomerular injury for any given reduction in BP as compared with both the $\mathrm{E}$ and $\mathrm{C}$ rats. This is demonstrated by the significant leftward shift of the slope of the relationship for nifedipine as compared with that for the control + enalapril-treated rats (N 1.34 $\pm 0.47, r=0.61, P<0.015 ; \mathrm{C}+\mathrm{E} 0.4 \pm 0.06, r=0.82$, $P<0.0001) ; \mathrm{N}$ vs. $\mathrm{C}+\mathrm{E}, P<0.02$. The increased susceptibility to glomerular injury in the nifedipine group is also demonstrated by a comparison of selected nifedipine- and enalapriltreated animals in which approximately similar BP control had been achieved. If the seven animals in the enalapril group with the highest overall averaged systolic $\mathrm{BP}(>110 \mathrm{mmHg}$; range $112-125 \mathrm{mmHg}$ ) are compared with the seven nifedipinetreated animals with the lowest overall averaged BP (range $113-132 \mathrm{mmHg}$ ), the BP was not significantly different between these selected groups $(118 \pm 2.4$ in the enalapril group vs. $125 \pm 2.9 \mathrm{mmHg}$ in the nifedipine group, $P>0.05$ ). However, both glomerular injury ( $3 \pm 1$ in the enalapril vs. $20 \pm 8 \%$ in the nifedipine group) and proteinuria ( $18 \pm 3.6$ in the enalapril vs. $61 \pm 19.5 \mathrm{mg} / 24 \mathrm{~h}$ in the nifedipine group) were significantly greater in the nifedipine-treated animals $(P<0.05$ for both comparisons). Fig. 6 illustrates the poor correlation of glomerular volume with the percentage of glomeruli exhibiting injury in individual animals $(r=0.31, P>0.05)$.

Autoregulatory studies. Table II provides the baseline data, $\mathrm{S}_{\mathrm{Cr}}$ at $3 \mathrm{~d}$, and the final $\mathrm{S}_{\mathrm{Cr}}, \mathrm{RBF}$, and GFR data for the animals undergoing autoregulatory studies. As for the radiotelemetry studies, no significant differences were present between the groups except for significantly lower final body weight in the nifedipine group. At 3 wk after renal ablation, both the kidney weights and glomerular volume showed no significant differences between groups.

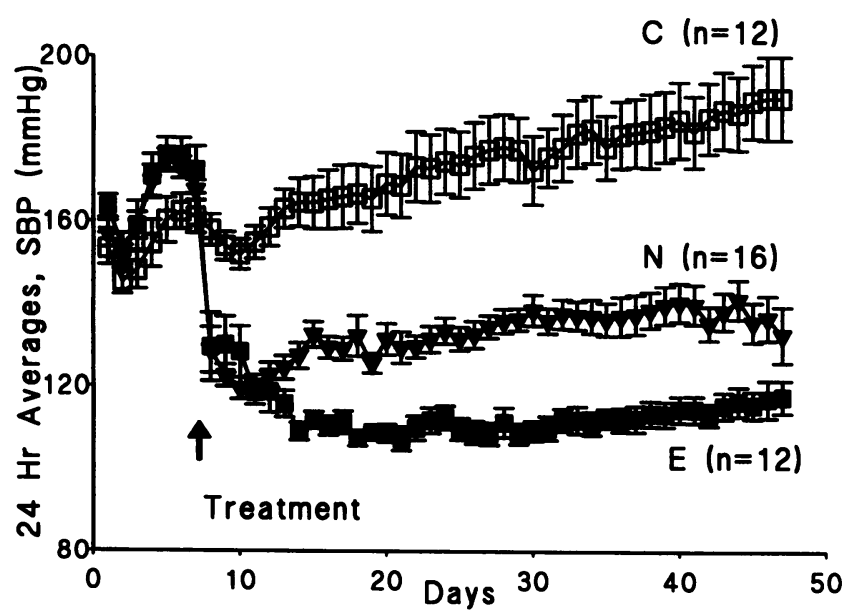

Figure 1. The course of systolic BP (24-h averages) over $7 \mathrm{wk}$ in the three groups. All rats underwent $\sim 5 / 6$ renal ablation (right uninephrectomy + infarction of $\sim 2 / 3$ of the left kidney). After $7 \mathrm{~d}(\uparrow)$, control rats received no treatment $(C)$, enalapril $50 \mathrm{mg} /$ liter of drinking water $(E)$, or $0.25 \%$ nifedipine in rat chow $(N)$. BP was radiotelemetrically recorded at 10-min intervals from the day of renal ablation surgery to killing $\sim 7 \mathrm{wk}$ later.

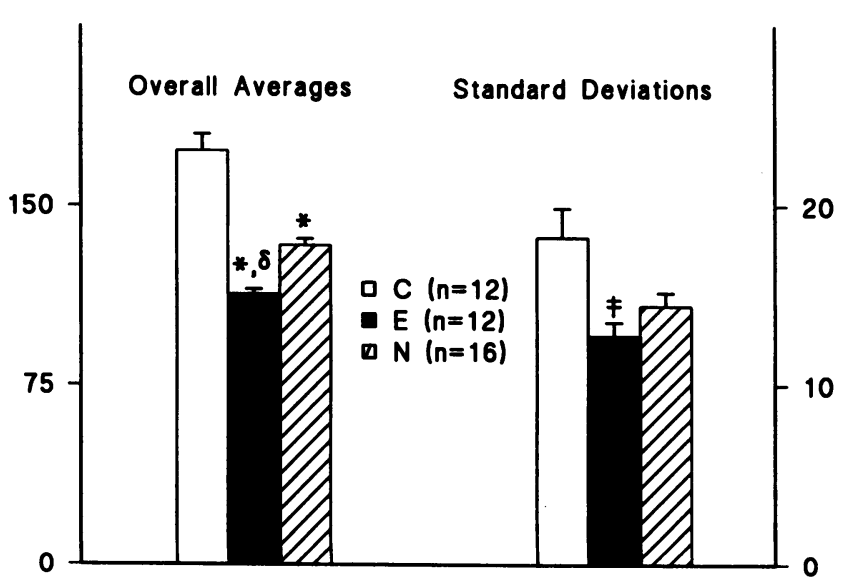

Figure 2. Overall averaged systolic BP $(\mathrm{mmHg})$ after day 7 in $\sim 5 / 6$ renal ablated rats who had received no treatment $(C), 0.25 \%$ nifedipine in rat chow $(N)$, or enalapril $50 \mathrm{mg} /$ liter of drinking water. BP was recorded radiotelemetrically at 10 -min intervals, and the overall averaged BP represents the mean of all BP readings from the day 7 to killing. The standard deviation of the overall averaged pressures, an index of the amplitude of BP fluctuations in the three groups, is also shown. ${ }^{*} P<0.001$ vs. $\mathrm{C} ;{ }^{\delta} P<0.01$ vs. $\mathrm{N} ;{ }^{\ddagger} P<0.05$ vs. $\mathrm{C}$. 

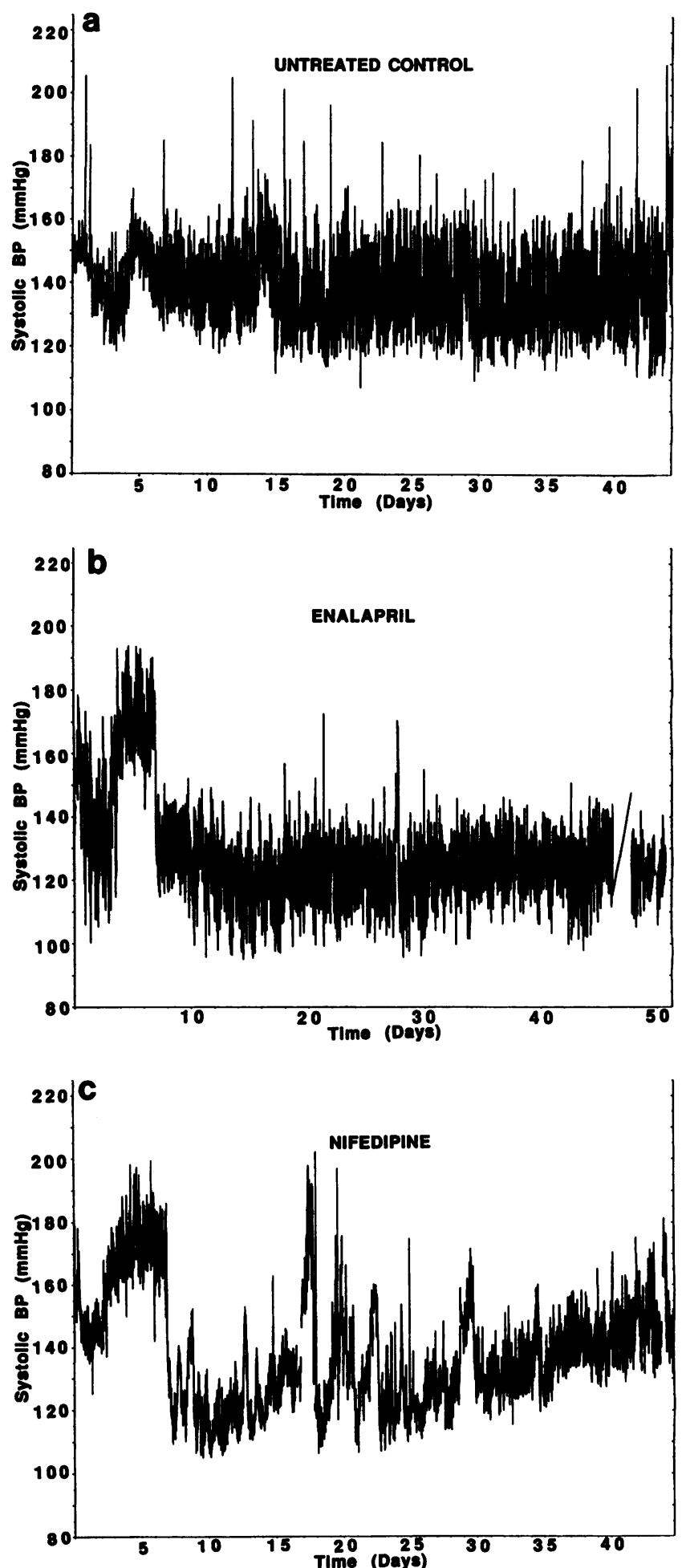

Figure 3. Illustrations of the course of systolic BP recorded every 10 min for $\sim 7 \mathrm{wk}$, in a rat with $\sim 5 / 6$ ablation from each of the three groups: (a) untreated control; $(b)$ enalapril; and $(c)$ nifedipine.

The data for protein excretion and glomerular injury at 3 wk for the rats that underwent autoregulatory studies are presented in Fig. $4 b$. In contrast to the 7-wk data which demonstrated no significant difference in protein excretion between the nifedipine-treated and control rats, at 3 wk proteinuria was significantly less in both enalapril $(P<0.01)$ and nifedipine
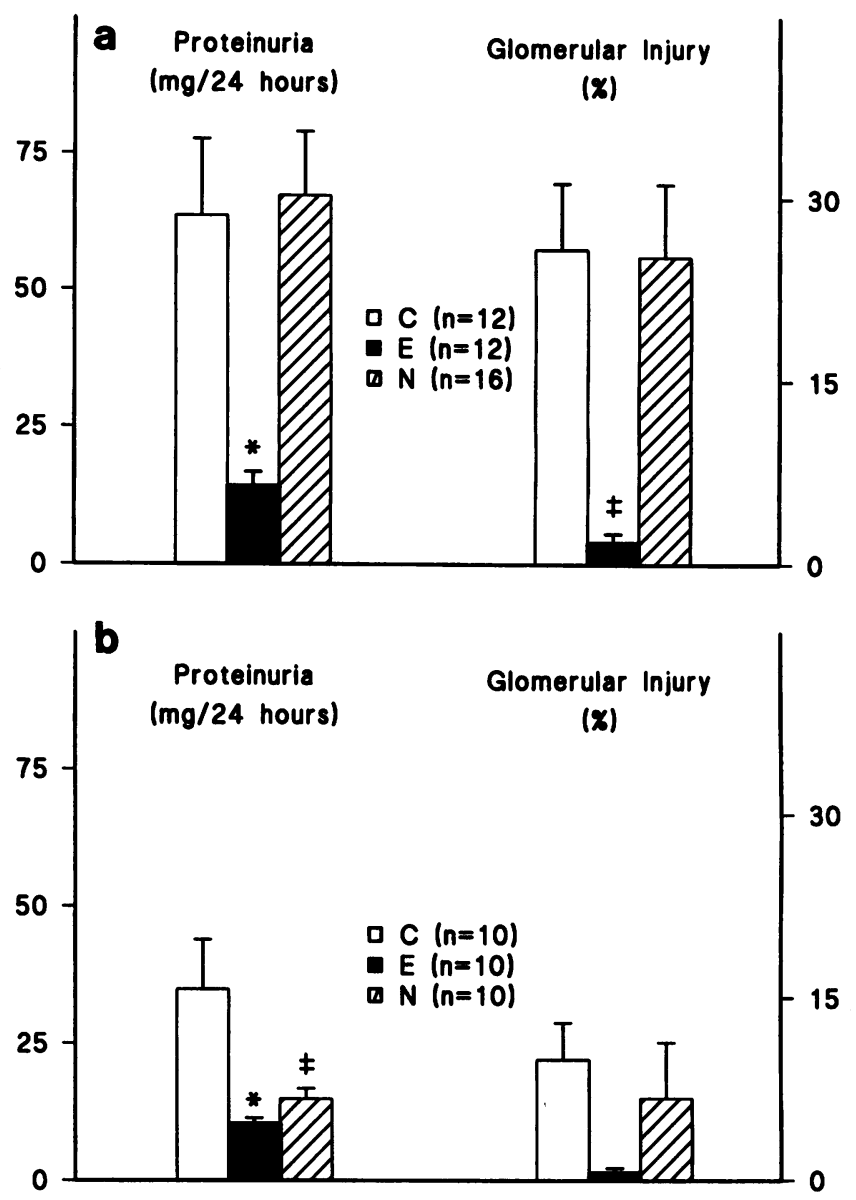

Figure 4. Proteinuria and percentage of glomerular injury in the three groups of rats with $\sim 5 / 6$ renal ablation. Untreated controls $(C)$; enalapril $(E)$; and nifedipine $(N)$. (a) at 7 wk in animals undergoing radiotelemetry studies and $(b)$ at $3 \mathrm{wk}$ in animals undergoing autoregulatory studies. At 7 wk: ${ }^{*} P<0.01$ vs. $\mathrm{C}$ and $\mathrm{N} ;{ }^{\ddagger} P<0.001$ vs. $\mathrm{C}$ and $\mathrm{N}$. At 3 wk: ${ }^{*} P<0.01$ vs. $\mathrm{C} ;{ }^{\ddagger} P<0.05$ vs. $\mathrm{C}$.

$(P<0.05)$ rats as compared with untreated animals. However, the glomerular injury data were qualitatively similar to those observed at 7 wk. Even as early as 3 wk after renal ablation, similar glomerular injury was present in both the nifedipine and control rats, but enalapril-treated rats had significantly less glomerular injury $(P<0.01)$.

The results of the RBF autoregulation studies are shown in Fig. 7. All three groups demonstrated significant impairment in RBF autoregulation, i.e., there were significant changes in RBF with each change in renal perfusion pressure. The autoregulatory ability was impaired to a similar degree in control and enalapril rats as indicated by the lack of significant differences in the calculated autoregulatory indices between them. In contrast, RBF autoregulation was further impaired in the nifedipinetreated rats and was significantly worse than in the control and enalapril-treated rats as indicated by the significantly higher autoregulatory indices in this group. The autoregulatory indices of $\sim 1.0$ indicate a complete loss of autoregulatory responses in the resistance vessels of the nifedipine-treated group.

\section{Discussion}

Hypertension is a consistent feature of the RK model when infarction of renal tissue is used to reduce renal mass $(2,4$, 


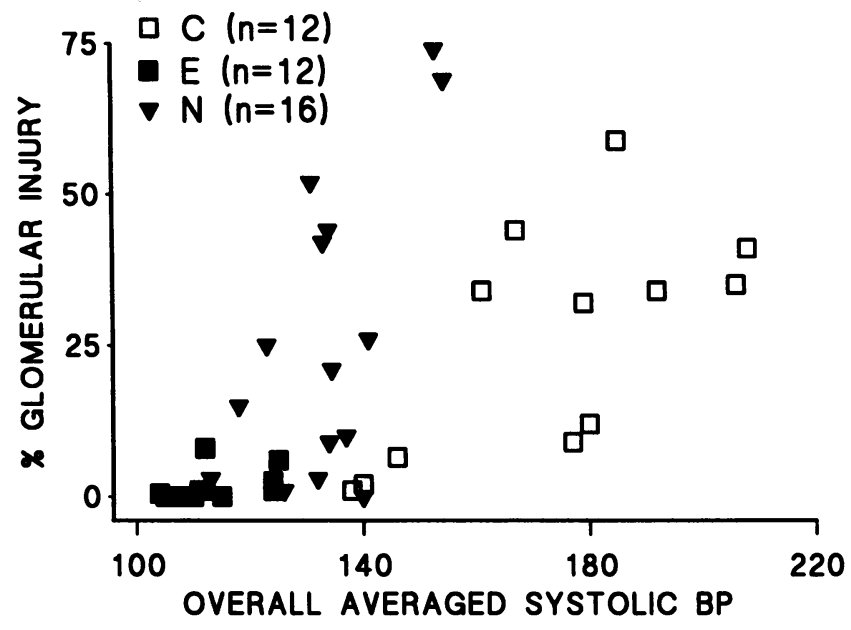

Figure 5. Correlation of percentage of glomeruli with injury (segmental sclerosis and/or necrosis) at time of killing in individual rats with $\sim 5$ / 6 ablation from all three groups with their overall averaged systolic BP for the final 6 wk (the mean of all BP readings, radiotelemetrically recorded every $10 \mathrm{~min}$, after the first $7 \mathrm{~d}$ ). After the first $7 \mathrm{~d}$, the rats had received no treatment $(C)$, enalapril $(E)$, or nifedipine $(N)$. The correlation coefficient $(r)$ for all rats was $0.57, P<0.0001$. But as can be noted, the slope of the relationship is different for $\mathrm{C}+\mathrm{E}$ rats than for $\mathrm{N}$ rats. For $\mathrm{C}+\mathrm{E}$ rats, the slope was $0.4 \pm 0.06, r=0.82, P$ $<0.0001$; and for $\mathrm{N}$ rats: slope, $1.34 \pm 0.47, r=0.61, P<0.015$. The slopes are significantly different from each other, $P<0.02$.

24). Substantial evidence also supports a dominant role of hypertensive mechanisms in the pathogenesis of the progressive glomerulosclerosis observed in the RK model $(4,5,7-11,24)$. The preglomerular vasodilation characteristic of reduced renal mass states has been demonstrated to result in an enhanced transmission of systemic pressures to the renal microvasculature as evidenced by an increase in $P_{G C}(3,4,9)$. The barotrauma associated with the resultant glomerular capillary hypertension is postulated to eventually result in glomerulosclerosis $(4,5$, 7-11). Nevertheless, several studies have documented that equivalent BP reductions with different antihypertensive agents do not confer comparable glomeruloprotection $(6,9,12-14)$. This has led some investigators to question the hypertensive pathogenesis of glomerulosclerosis in the RK model $(6,13$, 14), while others have postulated that glomeruloprotection by antihypertensives may depend on mechanisms other than or in addition to systemic $\mathrm{BP}$ reduction $(9,11,15,19)$. For these conclusions to be valid, it is critical that the achievement of equivalent BP control by different antihypertensive agents can be confidently assured. However, given the marked spontaneous lability of BP in rats with remnant kidneys documented by radiotelemetry in the present and previous studies $(20,21)$, it is extremely unlikely that the periodic tail-cuff BP measurements can achieve this goal. Indeed, our recent telemetric studies have shown that the superior glomeruloprotection afforded by enalapril as compared with triple therapy regimens in this model is largely a consequence of the superior and more consistent antihypertensive effects of enalapril which are not detected by the conventional tail-cuff methodology (21). Similarly, significant differences in the antihypertensive efficacies of nifedipine and enalapril were observed by radiotelemetry in this study, using dosages which have previously been reported to provide equivalent BP control by the tail-cuff method (19). While both

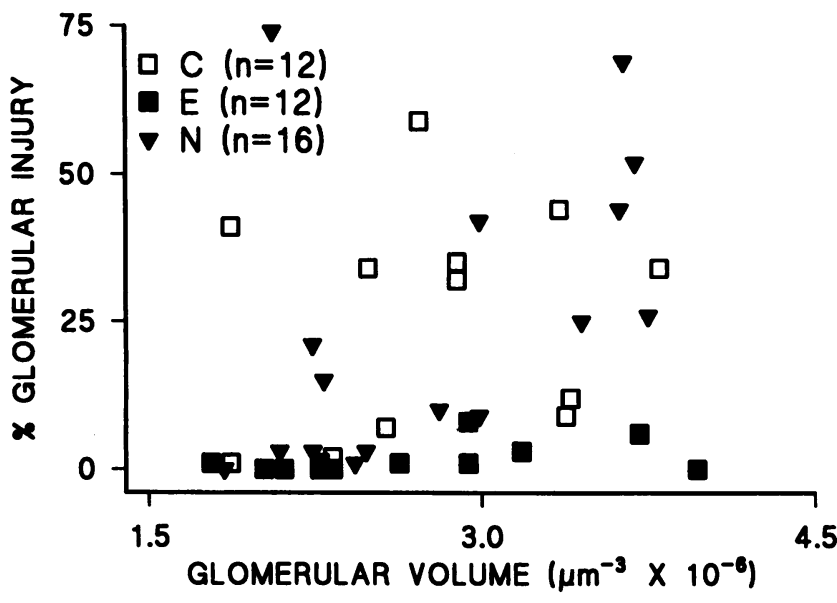

Figure 6. Correlation of percentage of glomeruli with injury (focal and segmental sclerosis and/or necrosis) at time of killing in individual rats with $\sim 5 / 6$ ablation from all three groups with the mean glomerular volume. After the first $7 \mathrm{~d}$, the rats had received no treatment $(C)$, enalapril $(E)$, or nifedipine $(N)$. For all animals $r=0.31, P>0.05$.

drugs significantly lowered BP, enalapril was more effective in the dosages used. In addition, a greater interanimal variability with respect to BP control was seen in the nifedipine group as compared with the more uniform BP reductions in the enalapril group. Both nifedipine and enalapril reduced the absolute amplitude of BP fluctuations as indicated by the smaller standard deviations of pressures in individual animals.

While BP reduction in enalapril-treated rats resulted in the expected and proportionate glomeruloprotection, the nifedipinetreated animals as a group failed to achieve such glomeruloprotection despite a significant reduction in BP when compared with the untreated control rats. Nevertheless, a strong correlation was still observed between BP and glomerular injury in individual rats in the nifedipine group $(r=0.61, P<0.015)$, consistent with BP-dependent mechanisms of glomerular injury. The explanation for the lack of glomeruloprotection in this group as a whole, despite significant BP reduction, is provided by the leftward shift of the slope of the relationship between overall averaged systolic BP and glomerular injury. As a result of this shift, more glomerular injury is sustained at any given BP elevation in nifedipine-treated rats. The steeper slope in the nifedipine group suggests that either there is a greater pressure transmission to the glomerular capillaries for any given systemic BP or that the glomeruli in the nifedipine-treated animals are more susceptible to injury for any given pressure transmission.

The conventional method to assess pressure transmission to the glomerular capillaries has been by the measurement of $P_{G C}$ during micropuncture studies $(4,6,9,13,17-19)$. The absolute increase in $\mathrm{P}_{\mathrm{GC}}$ is considered to be a direct index of the magnitude of the enhanced pressure transmission. The contribution of afferent arteriolar dilatation and or relative efferent arteriolar constriction to the elevation of $P_{G C}$ is additionally assessed during such studies. However, such studies with CCB in the RK model have provided variable and contradictory results. Although in normal rats with intact renal mass $(28,29)$ CCB predominantly cause afferent arteriolar dilatation and are thereby expected to favor increased glomerular pressure transmission, such effects have usually not been demonstrable in the RK model $(16,18,19)$. Effects on systemic $B P, P_{G C}$, and 
Table II. Renal Functional and Structural Parameters for Autoregulatory Studies

\begin{tabular}{|c|c|c|c|c|c|c|c|c|c|c|}
\hline & \multicolumn{3}{|c|}{ Initial } & \multirow{2}{*}{$\frac{3 \mathrm{~d}}{\mathrm{~S}_{\mathrm{cr}}}$} & \multicolumn{6}{|c|}{ Final (3 wk) } \\
\hline & Body wt & $\mathrm{s}_{\mathrm{cr}}$ & Proteinuria & & Body wt & $\mathrm{S}_{\mathrm{cr}}$ & GFR & RBF & Kidney wt & $\mathrm{v}_{\mathrm{G}}$ \\
\hline & grams & $m g / d l$ & $m g / 24 h$ & $m g / d l$ & grams & $m g / d l$ & $\mathrm{~m} / \mathrm{min} / \mathrm{kg}$ & $\mathrm{m} / \mathrm{min} / \mathrm{kg}$ & grams $/ k g$ & $\mu m^{-3} \times 10^{-6}$ \\
\hline $\mathrm{C}(n=10)$ & $233 \pm 5.7$ & $0.27 \pm 0.02$ & $3.6 \pm 0.6$ & $0.89 \pm 0.10$ & $292 \pm 15$ & $0.78 \pm 0.09$ & $3.3 \pm 0.5$ & $27.2 \pm 3.9$ & $8.8 \pm 0.4$ & $2.0 \pm 0.1$ \\
\hline $\mathrm{E}(n=10)$ & $231 \pm 6.3$ & $0.34 \pm 0.03$ & $3.1 \pm 0.6$ & $0.98 \pm 0.07$ & $265 \pm 7.4$ & $0.64 \pm 0.03$ & $3.9 \pm 0.3$ & $33.3 \pm 2.4$ & $8.0 \pm 0.4$ & $2.1 \pm 0.2$ \\
\hline $\mathrm{N}(n=10)$ & $237 \pm 5.9$ & $0.31 \pm 0.04$ & $2.5 \pm 0.3$ & $0.82 \pm 0.05$ & $250 \pm 9.7 *$ & $0.64 \pm 0.07$ & $4.3 \pm 0.4$ & $30.9 \pm 1.5$ & $8.0 \pm 0.4$ & $1.9 \pm 0.2$ \\
\hline
\end{tabular}

After baseline studies, all rats underwent $\sim 5 / 6$ ablation and underwent autoregulatory studies after $3 \mathrm{wk}$. After the first $7 \mathrm{~d}$, rats were left untreated (C) or received nifedipine $(\mathrm{N})$ or enalapril $(\mathrm{E}) .{ }^{*} P<0.05$ vs. C.

glomeruloprotection have also been variable and without a consistent relationship between these effects. For instance, longterm verapamil administration has been reported to provide glomeruloprotection (15) as well as to cause worsening of glomerular injury (17), in both cases without a significant reduction in systemic BP. The data are similarly conflicting with the dihydropyridine CCB. Although unlike verapamil, BP reductions comparable with those observed with converting enzyme inhibitors were achieved with both nifedipine (19) and felodopine (12); significant glomeruloprotection was only obtained with nifedipine, in spite of a persistently elevated $P_{G C}$.

Normally, the autoregulatory vasoconstrictor responses of the preglomerular vasculature provide the primary protection against increases in systemic pressure (episodic or sustained) from being transmitted to glomerular capillaries (30-32). In view of the fundamental spontaneous lability of BP in the unanesthetized state that has now been documented for most spe-

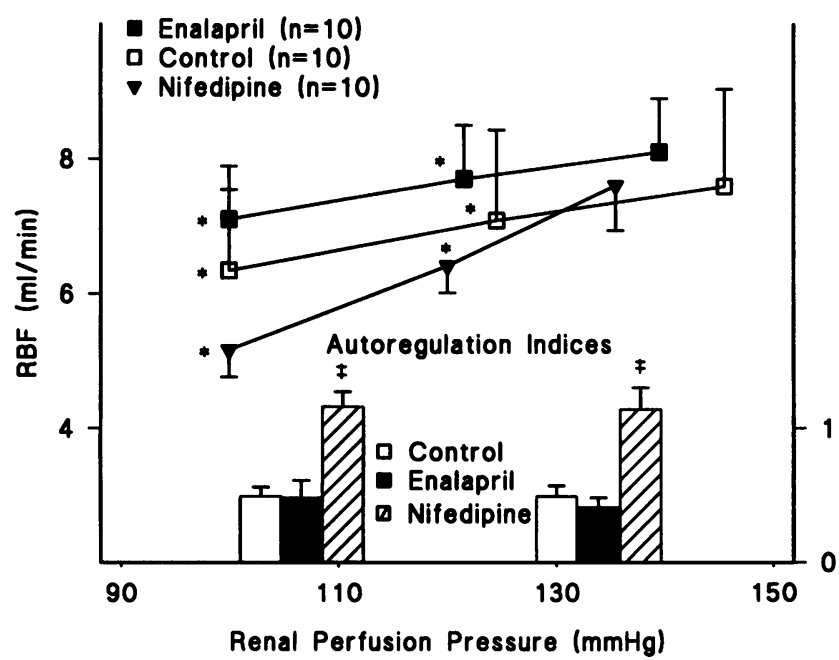

Figure 7. RBF autoregulatory studies in the three groups of rats who had undergone $\sim 5 / 6$ renal ablation 3 wk before. After day 7 , the rats were untreated or received enalapril or nifedipine. Graded changes in RPP were produced by aortic miniclamps and resulted in significant changes in RBF with every change in RPP, $* P<0.01$ repeated measures ANOVA. The bottom of the figure shows the calculated AIs for changes in RBF for each change in perfusion pressure in the three groups. An $\mathrm{AI}$ of 0 indicates perfect autoregulation, while an $\mathrm{AI}$ of $\sim 1.0$ indicates resistance vessels acting as passive conducts without autoregulatory responses. The AI were significantly greater in $\mathrm{N}$ vs. $\mathrm{C}$ or $\mathrm{E}$ groups, ${ }^{\ddagger} P$ $<0.01$. cies including humans (32-35), the efficiency of the autoregulatory responses to such fluctuations in BP is likely to be the primary determinant of the degree to which these fluctuations are transmitted to glomerular capillaries $(20,21,31,32)$. These autoregulatory responses have been demonstrated to be impaired in the remnant kidneys of untreated rats in these and previous studies $(5,8,22,24,36,37)$. Such impairment is expected to cause a lability of $\mathrm{P}_{\mathrm{GC}}$ in the conscious state, qualitatively similar to that observed for the systemic pressures. Micropuncture studies have supported this concept by demonstrating parallel changes in $P_{\mathrm{GC}}$ in remnant kidneys in response to either mechanically or pharmacologically produced changes in arterial pressure $(18,36,38)$. Therefore, pressure transmission to the glomeruli is likely to be a much more dynamic process than can be inferred from conventional measurements of BP and/or $\mathrm{P}_{\mathrm{GC}}$ which may not be representative of glomerular transmission of ambient systemic pressures in conscious rats with remnant kidneys. This may account for the inconsistent correlations of such measurements with glomeruloprotection after treatment with antihypertensive regimens including calcium channel blockers $(6,9,13,15,17,19)$. However, it is important to note that the renal autoregulatory responses, although impaired, were nevertheless still demonstrable in the control untreated animals (AI 0.5). Such impairment was not additionally affected by enalapril, but nifedipine essentially abolished the autoregulatory responses, resulting in the preglomerular vessels becoming passive conduits (AIs 1.0). The deleterious effects of nifedipine on renal autoregulatory ability would be expected to further exaggerate the transmission of labile systemic pressures to glomerular capillaries and provide an explanation for the adversely altered slope of the relationship between systemic BP and glomerular injury in the nifedipine-treated animals in this study. These observed effects of nifedipine on renal autoregulation in the RK model are consistent with the dependence of the autoregulatory vasoconstrictive response on calcium entry (39, 40 ) and the ability of CCB to impair renal autoregulation in rats with intact renal mass $(28,41,42)$. Therefore, the attainment of glomeruloprotection with CCB may depend on the net balance between the protective BP lowering effects on the systemic vasculature and the deleterious pressure transmission enhancing effects on the preglomerular renal vasculature.

In addition to the degree of glomerular pressure transmission, glomerular hypertrophy has been postulated to be another important determinant of susceptibility to glomerular injury (6, $13,19,43,44)$. Although the precise basis for the increased susceptibility of hypertrophied glomeruli has not been established, it has been suggested that glomerular capillary enlarge- 
ment may magnify the deleterious effects of increased $P_{G C}$ on capillary wall tension based on the LaPlace law, (wall tension $=$ pressure $\times$ radius $)(8,19,45,46)$. Indeed Dworkin et al. (19) have postulated that nifedipine and enalapril provide glomeruloprotection by separate and independent effects on these two determinants of wall tension in the RK model. The protection by nifedipine in their study was attributed to the prevention of glomerular hypertrophy-associated increases in capillary radii, as $P_{G C}$ was not reduced by nifedipine. By contrast, enalapril was postulated to have provided protection by reducing $\mathrm{P}_{\mathrm{GC}}$. The glomerular hypertrophy response was not altered by enalapril. Unlike that study, ameliorative effects on glomerular hypertrophy were not observed with either nifedipine or enalapril in this study. Moreover, little correlation of glomerular injury with glomerular volume was observed in individual animals. The reasons for these differences in results are not clear. CCB have many additional biologic effects that have the potential of altering the expression of glomerular injury in the RK model $(47,48)$. These include inhibitory effects on platelet aggregation, modulation of mesangial traffic of macromolecules, altered cellular response to growth factors, and reduction of remnant nephron hypermetabolism. However, these effects would be expected to enhance glomeruloprotection and, therefore, cannot account for the present results.

In conclusion, these data provide further support for the concept that glomerular transmission of hypertension plays a predominant role in the pathogenesis of progressive glomerular injury in reduced renal mass states. Additionally these studies stress the critical importance of autoregulatory mechanisms in such transmission and suggest that antihypertensive agents, such as the CCB that adversely affect autoregulatory ability and thereby enhance pressure transmission, may not provide glomeruloprotection despite significantly reducing BP. Further studies will be needed to ascertain whether these effects of nifedipine are shared by other CCB as well as to establish the dose dependency of these effects on the systemic and renal resistance vasculature. It is possible that the inconsistent glomeruloprotective efficacy of CCB is due to the potential variability in their effects on these two vascular beds in individual animals, studies, and models $(12,15-19,49,50)$. It is also not known whether similar considerations also apply to vascular beds in other target organs. Nevertheless, these data do suggest the need to assure that BP is maintained well within the normotensive range when CCB are used in clinical settings characterized by an enhanced BP transmission such as chronic renal disease and/or diabetes $(4,10,51,52)$.

\section{Acknowledgments}

The authors thank Wanda Plott, Jue Ouyang, and Lisa Kelly for technical assistance and Martha Prado for secretarial assistance.

This research was supported by National Institutes of Health grant DK-40426.

\section{References}

1. Shimamura, T., and A. B. Morrison. 1975. A progressive glomerulosclerosis occurring in 5/6 nephrectomized rats. Am. J. Pathol. 79:95-106.

2. Purkerson, M. L., P. E. Hoffsten, and S. Klahr. 1976. Pathogenesis of the glomerulopathy associated with renal infarction in rats. Kidney Int. 9:407-417.

3. Hostetter, T. H., J. L. Olson, H. G. Rennke, M. A. Venkatachalam, and B. M. Brenner. 1981. Hyperfiltration in remnant nephrons: a potentially adverse response to renal ablation. Am. J. Physiol. 241:F85-F93.
4. Brenner, B. M. 1985. Nephron adaptation to renal injury or ablation. Am. J. Physiol. 249:F324-F337.

5. Bidani, A. K., M. M. Schwartz, and E. J. Lewis. 1987. Renal autoregulation and vulnerability to hypertensive injury in remnant kidney. Am. J. Physiol. 252:F1003-F1010.

6. Klahr, S., G. Schreiner, and I. Ichikawa. 1988. The progression of renal disease. N. Engl. J. Med. 318:1657-1666.

7. Olson, J. L., and R. H. Heptinstall. 1988. Non-immunologic mechanisms of glomerular injury. Lab. Invest. 59:564-578.

8. Bidani, A. K., K. D. Mitchell, M. M. Schwartz, L. G. Navar, and E. J. Lewis. 1990. Absence of glomerular injury or nephron loss in a normotensive rat remnant kidney model. Kidney Int. 38:28-38.

9. Anderson, S., H. G. Rennke, and B. M. Brenner. 1986. Therapeutic advantage of converting enzyme inhibitors in arresting progressive renal disease associated with systemic hypertension in the rat. J. Clin. Invest. 77:1993-2000.

10. Baldwin, D. S., and J. Neugarten. 1987. Hypertension and renal disease. Kidney Dis. 10:186-191.

11. Tolins, J. P., and L. Raij. 1990. Angiotensin converting enzyme inhibitors and progression of chronic renal failure. Kidney Int. 38:S118-S122.

12. Jackson, B., and C. I. Johnston. 1988. The contribution of systemic hypertension to progression of chronic renal failure in the rat remnant kidneys: effect of treatment with an angiotensin converting enzyme inhibitor or a calcium inhibitor. J. Hypertens. 6:495-501.

13. Yoshida, Y., T. Kawamaura, M. Ikoma, A. Fogo, and I. Ichikawa. 1989. Effects of antihypertensive drugs on glomerular morphology. Kidney Int. 36:626635.

14. Kakinuma, Y., T. Kawamura, T. Bills, T. Yoshioka, I. Ichikawa, and A Fogo. 1992. Blood pressure-independent effect of angiotensin inhibition on vascular lesions of chronic renal failure. Kidney Int. 42:46-55.

15. Harris, D. C., W. S. Hammond, T. J. Burke, and R. W. Schrier. 1987. Verapamil protects against progression of experimental chronic renal failure. Kidney Int. 31:41-46.

16. Pelayo, J. C., D. C. H. Harris, P. F. Shanley, G. J. Miller, and R. W. Schrier. 1988. Glomerular hemodynamic adaptations in remnant nephrons: effects of verapamil. Am. J. Physiol. 254:F425-F431.

17. Brunner, F. P., G. Thiel, M. Hermle, A. Bock, and M. J. Mihatsch. 1989. Long-term enalapril and verapamil in rats with reduced renal mass. Kidney Int. 36:969-977.

18. Anderson, S. 1991. Renal hemodynamic effects of calcium antagonists in rats with reduced renal mass. Hypertension (Dallas). 17:288-295.

19. Dworkin, L. D., J. A. Benstein, M. Parker, E. Tolbert, and H. D. Feiner 1993. Calcium antagonists and converting enzyme inhibitors reduce renal injury by different mechanisms. Kidney Int. 43:808-814.

20. Bidani, A. K., K. A. Griffin, M. Picken, and D. M. Lansky. 1993. Continuous telemetric blood pressure monitoring and glomerular injury in the rat remnant kidney model. Am. J. Physiol. 265:F391-F398.

21. Griffin, K., M. Picken, and A. Bidani. 1994. Radiotelemetric BP monitoring, antihypertensives and glomeruloprotection in remnant kidney model. Kidney Int. 46:1010-1018.

22. Griffin, K. A., A. K. Bidani, M. Picken, V. R. Ellis, and P. C. Churchill 1992. Prostaglandins do not mediate impaired autoregulation or increased renin secretion in remnant rat kidneys. Am. J. Physiol. 263:F1057-F1062.

23. Griffin, K. A., A. K. Bidani, J. Ouyang, V. Ellis, M. Churchill, and P. C. Churchill. 1993. Role of endothelium-derived nitric oxide in hemodynamic adaptations after graded renal mass reduction. Am. J. Physiol. 264:R1254-R1259.

24. Griffin, K. A., M. Picken, and A. K. Bidani. 1994. Method of renal mass reduction is a critical modulator of subsequent hypertension and glomerular injury. J. Am. Soc. Nephrol. 4:2023-2031.

25. Weibel, E. R. 1979. Sterological Methods: Practical Methods for Biological Morphometry. Vol. 1. Academia, London. 51-57.

26. Semple, S. J. G., and H. E. deWardener. 1959. Effect of increased renal venous pressure on circulatory "autoregulation" of isolated dog kidneys. Circ. Res. 7:643-648.

27. Wallenstein, S., C. L. Zucker, and J. Fleiss. 1980. Some statistical methods useful in circulation research. Circ. Res. 47:1-9.

28. Loutzenhiser, R., and M. Epstein. 1985. Effects of calcium antagonists on renal hemodynamics. Am. J. Physiol. 249:F619-F629.

29. Carmines, P. K., and L. G. Navar. 1989. Disparate effects of Ca channel blockade on afferent and efferent arteriolar responses to ANG II. Am. J. Physiol. 256:F1015-F1020.

30. Ofstad, J., and K. Aukland. 1985. Renal circulation. In The Kidney, Physiology and Pathophysiology. D. W. Seldin and G. Giebisch, editors. Raven Press, New York. 471-496.

31. Daniels, F. H., W. J. Arendshorst, and R. G. Roberds. 1990. Tubuloglomerular feedback and autoregulation in spontaneously hypertensive rats. Am. J. Physiol. 258:F1479-F1489.

32. Holstein-Rathlou, N. H., A. J. Wagner, and D. J. Marsh. 1991. Tubuloglomerular feedback dynamics and renal blood flow autoregulation in rats. Am. J. Physiol. 260:F53-F68.

33. Akselrod, S., D. Gordon, F. A. Ubel, D. C. Shannon, A. C. Barger, and 
R. J. Cohen. 1981. Power spectrum analysis of heart rate fluctuation: a quantitative probe of beat-to-beat cardiovascular control. Science (Wash. DC). 213:220-223.

34. Pagani, M., F. Lombardi, S. Guzzetti, O. Rimoldi, R. Furlan, P. Pizzinelli, G. Sandrone, G. Malfatio, S. Dell'Orto, E. Piccaluga, et al. 1986. Power spectral analysis of heart rate and arterial pressure variabilities as a marker of sympathovagal interaction in man and conscious dogs. Circ. Res. 59:178-193.

35. Parati, G., S. Omboni, M. DiRienzo, A. Frattola, F. Albini, and G. Mancia. 1992. Twenty-four hour blood pressure variability: clinical implications. Kidney Int. 41:S24-S28.

36. Pelayo, J. C., and J. Y. Westcott. 1991. Impaired autoregulation of glomerular capillary hydrostatic pressure in the rat remnant nephron. J. Clin. Invest. 88:101-105.

37. Bidani, A. K., K. A. Griffin, W. Plott, and M. M. Schwartz. 1994. Renal ablation acutely transforms "benign" hypertension to "malignant" nephrosclerosis in hypertensive rats. Hypertension (Dallas). 24:309-316.

38. Yoshioka, T., H. Shirage, Y. Yoshida, A. Fogo, A. D. Glick, W. M. Deen, J. R. Hoyer, and I. Ichikawa. 1988. "Intact nephrons" as the primary origin of proteinuria in chronic renal disease. J. Clin. Invest. 82:1614-1623.

39. Cohen, A. J., and J. C. S. Fray. 1982. Calcium ion dependence of myogenic renal plasma flow autoregulation: evidence from the isolated perfused rat kidney. J. Physiol. (Lond.). 330:449-460.

40. Churchill, P. C. 1990. Calcium, calcium antagonists, and renin secretion. In Calcium Antagonists and the Kidney. M. Epstein and R. Loutzenhiser, editors. Hanley \& Belfus, Philadelphia. 127-154.

41. Navar, L. G., W. J. Champion, and C. E. Thomas. 1986. Effects of calcium channel blockade on renal vascular resistance responses to changes in perfusion pressure and angiotensin-converting enzyme inhibition in dogs. Circ. Res. 58:874881.
42. Kvam, F. I., B. M. Iverson, and J. Ofstad. 1994. Glomerular capillary pressure is dependent on systemic blood pressure during treatment with calcium channel blockers. J. Am. Soc. Nephrol. 5:543a. (Abstr.)

43. Yoshida, Y., A. Fogo, and I. Ichikawa. 1989. Glomerular hemodynamic changes vs. hypertrophy in experimental glomerular sclerosis. Kidney Int. 35:654660 .

44. Miller, P. L., H. G. Rennke, and T. W. Meyer. 1991. Glomerular hypertrophy accelerates hypertensive glomerular injury in rats. Am. J. Physiol. 261:F459F465.

45. Daniels, B. S., and T. H. Hostetter. 1990. Adverse effects of growth in the glomerular microcirculation. Am. J. Physiol. 258:F1409-F1416.

46. Wolf, A. V. 1952. Demonstration concerning pressure-tension relations in various organs. Science (Wash. DC). 115:243-244.

47. Epstein, M. 1993. Calcium antagonists and the kidney: future therapeutic perspectives. Am. J. Kidney Dis. 21 (Suppl. 3):16-25.

48. Haller, H. 1993. Calcium antagonists and cellular mechanisms of glomerulosclerosis and atherosclerosis. Am. J. Kidney Dis. 21 (Suppl. 3):26-31.

49. Bakris, G. L. 1991. Renal effects of calcium antagonists in diabetes mellitus. An overview of studies in animal models and in humans. Am. J. Hypertens. 4:487S-493S.

50. Anderson, S., H. G. Rennke, and B. M. Brenner. 1992. Nifedipine versus fosinopril in uninephrectomized diabetic rats. Kidney Int. 41:891-897.

51. Zatz, R., T. W. Meyer, H. G. Rennke, and B. M. Brenner. 1985. Predominance of hemodynamic rather than metabolic factors in the pathogenesis of diabetic glomerulopathy. Proc. Natl. Acad. Sci. USA. 82:5963-5967.

52. Morgensen, C. E. 1982. Long-term antihypertensive treatment inhibiting progression of diabetic nephropathy. BMJ (Br. Med. J.). 285:685-688. 